\title{
Anomalous Spreading of Power-Law Quantum Wave Packets
}

\author{
Fabrizio Lillo and Rosario N. Mantegna \\ Istituto Nazionale per la Fisica della Materia, Unità di Palermo \\ and \\ Dipartimento di Energetica ed Applicazioni di Fisica, Università di Palermo, Viale delle Scienze, I-90128, Palermo, Italia
}

\begin{abstract}
We introduce power-law tail quantum wave packets. We show that they can be seen as eigenfunctions of a Hamiltonian with a physical potential. We prove that the free evolution of these packets presents an asymptotic decay of the maximum of the wave packets which is anomalous for an interval of the characterizing power-law exponent. We also prove that the number of finite moments of the wave packets is a conserved quantity during the evolution of the wave packet in the free space.
\end{abstract}

03.65.-w, 05.40.Fb

Power-law probability density functions [1] are receiving a lot of attention in different research fields [2,3]. Stochastic processes with power-law distributions may or may not be characterized by a typical scale in time and/or in the size of the random variable. One implication of the absence of a typical scale is the divergence of the variance of the distribution. Examples of phenomena without a typical scale are observed in physical systems at the critical state [4], in self-organized [5] and in complex systems [6]. Considering few-body quantum systems - (i) scale free power-law processes have been observed in a quantum system by investigating and modeling experiments of velocity selective coherent population trapping [7,8] and (ii) the power-law temporal growth of the moments of a wave packet has been investigated in quantum systems with fractal energy spectra and eigenfunctions such as the Harper model [9.10]. Spatial power-law wave functions have not been considered within the framework of non-relativistic quantum mechanics. The probabilistic interpretation of the wave function and the recent results on power-law distributions observed in physical systems motivates us to investigate the properties of powerlaw wave functions in quantum mechanics. In this letter we consider quantum wave packets with power-law tails. Specifically we focus on - (i) their physical properties. Namely the uncertainty product, the associated energy and the momentum distribution and (ii) the spreading of such quantum wave packets during the free evolution.

We define as Power-Law Tail Wave Packet (PLTWP) a wave function $\psi(x)$ describing a non-relativistic spinless particle in one dimension, which decreases with $x$ as

$$
|\psi(x)| \sim|x|^{-\alpha} \text {. }
$$

This class of wave packets is square-integrable only if $\alpha>1 / 2$. For the sake of simplicity, in this study we assume that wave function is real, positive and even. The study of quantum wave packets with wave function real or complex, uneven and with zeros is presented elsewhere [11]. One of the properties of power-law distributions is that only a finite number of moments of the variable are finite. In quantum theory this implies that the moments of position operator $\left\langle\hat{x}^{m}\right\rangle$ with $m \geq 2 \alpha-1$ are infinite. The lack of finite moments of $x$ has a counterpart in the properties in $k=0$ of Fourier transform $g(k)=F T[\psi(x)]$ which gives the amplitude probability distribution of momentum. We note that when $n<\alpha \leq n+1$, only the first $n-1$ derivatives of $g(k)$ exist in $k=0$. In the special case $1 / 2<\alpha \leq 1$ the Fourier transform $g(k)$ is infinite in $k=0$. The behavior of $g(k)$ when $k \simeq 0$ is described by two different series expansions depending on the value of $\alpha$. Specifically, when $\alpha$ is not an odd integer number

$$
g(k) \simeq a_{0}+a_{2} k^{2}+\ldots+a_{2 n} k^{2 n}+b|k|^{\alpha-1}+o\left(|k|^{\alpha}\right),
$$

where $2 n$ is the largest even integer number smaller than $\alpha-1$. When $\alpha=2 n+1$ the series expansion is

$$
g(k) \simeq a_{0}+a_{2} k^{2}+\ldots+a_{2 n} k^{2 n}+b k^{2 n} \log |k|+\ldots .
$$

The case $\alpha=1$ has a logarithmic divergence in $k=0$.

In spite of this behavior we wish to point out that all moments of the momentum operator (including the kinetic energy) of the particle $\left\langle\hat{p}^{m}\right\rangle(m \geq 1)$ are finite. In fact, a property of Fourier transform is

$$
F T\left[\psi^{(m)}(x)\right]=(-i k)^{m} g(k),
$$

where $\psi^{(m)}(x)$ indicates the $m$-th derivative of $\psi(x)$. This property is valid under the hypothesis that $\lim _{|x| \rightarrow \infty} \psi^{(r)}(x)=0$ for $r=0,1, \ldots, m-1$. Since the tails behave as $|x|^{-\alpha-m}, \psi^{(m)}(x)$ is absolutely integrable. By using Riemann-Lebesgue lemma [12], one can conclude that $\lim _{k \rightarrow \infty} k^{m} g(k)=0$ for all $m$.

A consequence of the finiteness of momentum moments is that the uncertainty in momentum $\Delta k$ is always finite for PLTWP. As mentioned above, when $\alpha \leq \frac{3}{2}$ the second moment of the position operator and thus the root mean square deviation $\Delta x$ of position are infinite. Therefore this kind of wave packets have an uncertainty product $\Delta x \Delta k$ which is infinite when $\alpha \leq \frac{3}{2}$. This physical property reflects the fact that for PLTWP with $\alpha \leq \frac{3}{2}$ a 
typical scale exists in momentum space whereas the wave packet is scale free in space.

Can a PLTWP be an eigenfunction of an Hamiltonian? To answer this question we note that each PLTWP can be related to a specific physical potential. The PLTWP is then an eigenfunction of the corresponding Hamiltonian. The equation providing the potential associated with a particle described by a wave function $\psi(x)$ is 13]

$$
U(x)=E+\frac{\hbar^{2}}{2 M} \frac{1}{\psi(x)} \frac{d^{2} \psi(x)}{d x^{2}},
$$

where $E$ is the eigenvalue of energy and $M$ is the mass of the particle. The shape of the potential depends on the local properties of the wave function. ¿From Eqs. (1) and (5) it is immediate to conclude that the potential associated with PLTWP behaves asymptotically as $x^{-2}$ [14]. As an illustrative example we present PLTWPs defined as

$$
\psi(x)=\frac{N}{\left(x^{2}+\gamma^{2}\right)^{\alpha / 2}},
$$

where $N$ is a suitable normalization constant and $\gamma$ is a scale parameter. The above family of quantum wave packets is related to the Student's $t$-distribution when $\alpha$ is integer. The associated potential is

$$
U(x)=\frac{\hbar^{2}}{2 M} \alpha \frac{x^{2}(1+\alpha)-\gamma^{2}}{\left(x^{2}+\gamma^{2}\right)^{2}},
$$

and is shown in Fig. 1. In this figure the eigenvalue $E$ is set equal to zero. The potential is a single well potential with two symmetrical confining barriers. The potential reaches a maximal value and then decreases asymptotically as $U(x) \sim x^{-2}$. A general property of this potential is that by increasing the value of $\alpha$ the depth of the potential well increases. From Fig. 1 it is clear that the associated potential does not present anomalies of any sort.

Hereafter we consider the properties of a PLTWP in the simplest case of dynamical evolution, namely the free wave evolution. We assume that a $t=0$ the wave packet in the free space has the asymptotic properties of Eq. (1). We focus our attention on the spreading of the wave packet. During the dynamical evolution in free space the wave function at time $t$ is given by

$$
\psi(x, t)=\frac{1}{\sqrt{2 \pi}} \int_{-\infty}^{\infty} g(k) e^{i\left(k x-\hbar t k^{2} / 2 M\right)} d k .
$$

We briefly recall that in the case of a Gaussian wave packet the amount of spreading of the wave packet can be quantified either by considering the time dependence of the position variance or by determining the time dependence of the maximum of the wave function. To quantify the amount of spreading of a free wave packet in the same way for PLTWP with finite or infinite variance we choose to focus our attention to the asymptotic behavior in time of the wave function in a specific position (for example $x=0)$. In the Gaussian case, the variance of $|\psi(x, t)|^{2}$ is asymptotically proportional to $t^{2}$ and the maximum of the wave function $|\psi(0, t)|^{2}$ decreases as $t^{-1}$ asymptotically. We will show in the following that this asymptotic behavior observed for Gaussian quantum packets is not universally observed in the free evolution of a quantum wave packet.

We consider here the free evolution of the maximum of the packet, which is described by Eq. (8) with $x=0$. It is possible to give an asymptotic expansion in time $t$ of integral of Eq. (8) using phase stationary method (see for example [15]). This method shows that the asymptotic expansion of $\psi(0, t)$ is determined by the dominant term of series expansion about the origin $(k=0)$ of $g(k)$ and by the function $-\hbar t k^{2} / 2 M$. The first term of the series expansion about $k=0$ of $g(k)$ is $g(k) \simeq Q|k|^{\lambda-1}$ for any value of $\alpha \neq 1$. By using phase stationary method [15], we know that when $0<\lambda<2$ it is possible to give an asymptotic expansion of integral of Eq. (8) with $x=0$ in the form

$$
\psi(0, t) \sim \frac{1}{\sqrt{2 \pi}} e^{-i \pi \lambda / 4} Q \Gamma\left(\frac{\lambda}{2}\right) \frac{1}{\beta^{\lambda / 2}},
$$

where $\beta=\hbar t / 2 M$. If $g(k)$ has a finite non-vanishing limit as $k$ goes to zero then $\lambda=1$ and we conclude that the maximum of the packet decreases asymptotically in time as $1 / \sqrt{t}$. This is the case, for example, of Gaussian wave packets cited above and, in general, of all commonest wave packets with finite FT in $k=0$. From Eqs. $(2,3)$ and (9) we conclude that this is also the case of PLTWP with $\alpha>1$. These packets show a customary asymptotic dynamics of the maximum. PLTWPs with $1 / 2<\alpha<1$ show a different behavior. In fact, in these cases $g(k)$ diverges in $k=0$ as $|k|^{\alpha-1}$ and, as a consequence $\lambda=\alpha<1$. Hence, form Eq. (9) we conclude that the maximum decreases asymptotically as $t^{-\alpha / 2}$. This is a maximum decrease, which is anomalous and slower with respect to the decrease observed in customary wave packets. The $t^{-1 / 2}$ behavior observed in Gaussian wave packets is interpreted in terms of the time evolution of a group of classical particles with a momentum dispersion $\Delta p$. A similar simple picture cannot explain the behavior observed for PLTWPs with $\alpha<1$ [16].

To illustrate the process of convergence of different wave packets to the expected asymptotic behavior we calculate the time evolution of the amplitude of wave packet at $x=0$ for three different cases. Specifically we consider a Gaussian wave packet and two PLTWPs of the class defined by Eq. (6) with $\alpha=3$ and $\alpha=0.75$. In Fig. 2 we show the numerical estimate of $|\psi(0, t)|$. In the figure is clear that the Gaussian and the PLTWP with $\alpha=3$ soon converge to the usual $1 / \sqrt{t}$ asymptotic behavior whereas the PLTWP with $\alpha=0.75$ slowly converges to the anomalous asymptotic behavior of $1 / t^{\alpha / 2}=1 / t^{0.375}$.

The case $\alpha=1$ cannot be handled with phase stationary method because of logarithmic divergence of $g(k)$ in 
$k=0$. Although we are not able to provide a general answer for the case $\alpha=1$, we are able to determine the asymptotic behavior in the specific case of a packet described by Eq. (6) with $\alpha=1$. For a such wave packet, the maximum decreases as $1 / \sqrt{t}$.

In order to obtain a more complete description of the free wave spreading, we consider the time evolution of the tails of the packet. We prove that the free wave packet evolution of a PLTWP conserves the tails. More precisely, if at $t=0$ the dominant term of asymptotic expansion of $\psi(x)$ is $c|x|^{-\alpha}$, at each subsequent time the asymptotic expansion of $\psi(x, t)$ will be dominated by $c|x|^{-\alpha}$. In this sense, the free evolution cannot change the asymptotic properties of the packet. We can visualize this property dividing the whole set of PLTWPs in classes such as each class is characterized by a value of $\alpha$. In other words, any packet belongs to the same class at any time during the free wave evolution. Since $\alpha$ determines the number of finite moments of operator $\hat{x}$, our result implies that position moments cannot became finite if they were infinite at $t=0$ and vice versa. We prove our statement as follows. Let us first consider Eq. (8) and look for the asymptotic expansion in $x$ of the FT of $f(k)=g(k) e^{-i \beta k^{2}}$. The asymptotic expansion of the FT of $f(k)$ is determined by its singularities [12]. By following Ref. [12], we say that $f(k)$ is singular in $k_{0}$ if one cannot differentiate $f(k)$ in $k_{0}$ any number of times. From properties of $g(k)$ of PLTWPs we conclude that $k=0$ is the only singularity for $f(k)$. In order to find the asymptotic expansion of Eq. (8), we construct a function $F(k)$ in such a way that $\tilde{f}(k) \equiv f(k)-F(k)$ has absolutely integrable $m$-th derivative in an interval including $k=0 . F(k)$ must be a linear combination of powers of $k$ and product of powers of $k$ and $\log k$ [12. Moreover the $m$-th derivative of $f(k)$ must be absolutely integrable in an interval from a real value to infinity. If all these hypothesis are verified, the FT of $f(k)$ is equal to the FT of $F(k)$ plus $o\left(|x|^{-m}\right)$. Depending on the specific value of $\alpha$ the regularizing function assumes a different form. In spite of this, our results do not depend on the specific value of $\alpha$. For the sake of simplicity, we present here the case with $2 n+1<\alpha<2 n+2$. The demonstration of cases for other value of $\alpha$ (specifically $2 n<\alpha<2 n+1, \alpha=2 n$ and $\alpha=2 n+1)$ are similar. In order to regularize the $2 n+1$-th derivative of $f(k)$ the natural choice is

$$
\tilde{f}(k)=g(k) e^{-i \beta k^{2}}-b|k|^{\alpha-1} .
$$

In fact the first $2 n+3$ derivatives of $\tilde{f}(k)$ are absolutely integrable in an interval including $k=0$. Therefore, we obtain the asymptotic expansion of $\psi(x, t)$ as

$$
\begin{array}{r}
\psi(x, t)=F T(F(k))+o\left(|x|^{-2 n-3}\right)= \\
c|x|^{-\alpha}+o\left(|x|^{-2 n-3}\right)
\end{array}
$$

which demonstrates our assertion.

As an illustrative example let us consider the free evolution of the PLTWP of Eq. (6) with $\alpha=2$ and $\gamma=1$.
The corresponding wave function has the form of the Cauchy distribution and it is possible to find the $\psi(x, t)$ analytically. In Fig. 3 we show the square modulus of the positive tail of the packet versus $x$ at different times in a log-log plot. The figure shows that the dominant term of the asymptotic expansion, i.e. $x^{-4}$, is the same at any time. The figure also shows that new terms of the asymptotic expansion become relevant at longer times and the region of asymptotic convergence moves towards larger values of $x$.

A connection between stochastic processes and quantum processes has been considered within the framework of stochastic mechanics [17]. Here we note that the free evolution of a quantum wave packet is closely related to a superdiffusive (ballistic) stochastic process. When a superdiffusive behavior is observed in classical 18 20 and quantum [21] processes, the central limit theorem does not apply and a general theoretical description is lacking. In our study we obtain two general conclusions in the problem of the free evolution of a PLTWP. The first concerns the temporal evolution of the maximum of the wave packet (which corresponds to the probability of return to the origin in stochastic processes). In the well-known case of a Gaussian wave packet (which has a fractional Brownian motion with exponent $h=1$ as the approximately corresponding stochastic process [22]) the variance is quadratic in time and the probability of return to the origin is inversely proportional to the time. Similarly, we find that the same behavior is asymptotically observed for the evolution of an even and real PLTWP when the exponent $\alpha$ is greater than one. Conversely, for values of $\alpha$ within the interval $1 / 2<\alpha<1$ we observe an anomalous behavior of the time evolution of the maximum of the wave packet. We are not able to interpret this unexpected behavior on a semiclassical basis. The second conclusion concerns the conservation during the quantum time evolution of the number of finite/infinite moments of the $t=0$ distribution. This behavior is peculiar to this quantum dynamics and could not be observed, for example, in stochastic processes obeying the central limit theorem.

We thank INFM and MURST for financial support. We wish to thank Giovanni Bonanno for help in numerical calculations.

[1] P. Lévy, Calcul des Probabilités (Gauthier-Villars, Paris, 1925).

[2] B. B. Mandelbrot, The Fractal Geometry of Nature (W.H. Freeman and Company, New York, 1982).

[3] M.F. Shlesinger, G. M. Zaslavsky and U. Frisch (Eds.), Lévy Flights and Related Topics in Physics (SpringerVerlag, Berlin and Heidelberg, 1995).

[4] H. E. Stanley, Introduction to Phase Transitions and 
Critical Phenomena (Oxford University Press, New York, 1971).

[5] P. Bak, C. Tang and K. Wiesenfeld, Phys. Rev. Lett. 57, 381 (1987).

[6] F. Mallamace and H. E. Stanley (Eds.), The Physics of Complex Systems (IOS Press, Amsterdam, 1997).

[7] F. Bardou et al., Phys. Rev. Lett. 72, 203 (1994).

[8] J. Reichel et al., Phys. Rev. Lett. 75, 4575 (1995).

[9] T. Geisel, R. Ketzmerick and G. Petschel, Phys. Rev. Lett. 66, 1651 (1991).

[10] R. Ketzmerick et al., Phys. Rev. Lett. 79, 1959 (1997).

[11] F. Lillo and R.N. Mantegna, (in preparation)

[12] M. J. Lighthill, Introduction to Fourier Analysis and Generalised Functions (Cambridge University Press, Cambridge, 1970).

[13] W. E. Lamb Jr. , Physics Today 22, No 4, 23 (1969).

[14] The power-law behavior of $\psi(x)$ is also observed for large values of $|x|$ for eigenfunctions with energy $E^{*}$ greater than $E$ provided that $|x| \ll \sqrt{\hbar^{2} \alpha(\alpha+1) / 2 M\left(E^{*}-E\right)}$. The existence of bound states and their properties depends on the specific form of $U(x)$ nearby $x=0$.

[15] F. W. J. Olver, Asymptotics and Special Functions (Academic Press, New York and London, 1974).

[16] The analogy between the Wigner function and the corresponding classical probability distribution in the phase space cannot be used for PLTWPs because the Wigner function of such quantum states becomes negative in some regions of the phase space (for a proof see Ref. [11]). Hence a simple analogy cannot be pursued in spite of the fact that the dynamical equation for the Wigner function is identical with the classical Liouville equation in the free evolution.

[17] F. Guerra, Phys. Rep. 77, 263 (1981).

[18] M. Cassandro and G. Jona-Lasinio, Advances in Physics 27, 913 (1978).

[19] M.F. Shlesinger, G. M. Zaslavsky and J. Klafter, Nature 363, 31 (1993).

[20] G.M. Zaslavsky, M. Edelman, and B.A. Niyazov, Chaos 7, 159 (1997).

[21] B. Sundaram and G.M. Zaslavsky, Phys. Rev. E 59, 7231 (1999).

[22] B. B. Mandelbrot and J. W. van Ness, SIAM Review 10, 422 (1968).

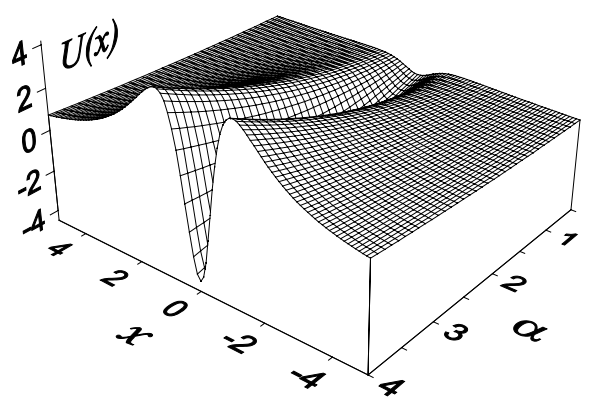

FIG. 1. Potential profile, Eq. (7), associated with the class of power-law tail wave packets of Eq. (6). Here we show a region of $\alpha$ values ranging from the minimal allowed value $\alpha=1 / 2$ (excluded) to the value $\alpha=4$. In the entire interval $\alpha>1 / 2$, the potential does not show any pathological behavior. In our calculation we set $\gamma=1$. The potential $U(x)$ is given in units of $\hbar^{2} / 2 M$. The PLTWP is the eigenfunction of the corresponding Hamiltonian with eigenvalue $E=0$.

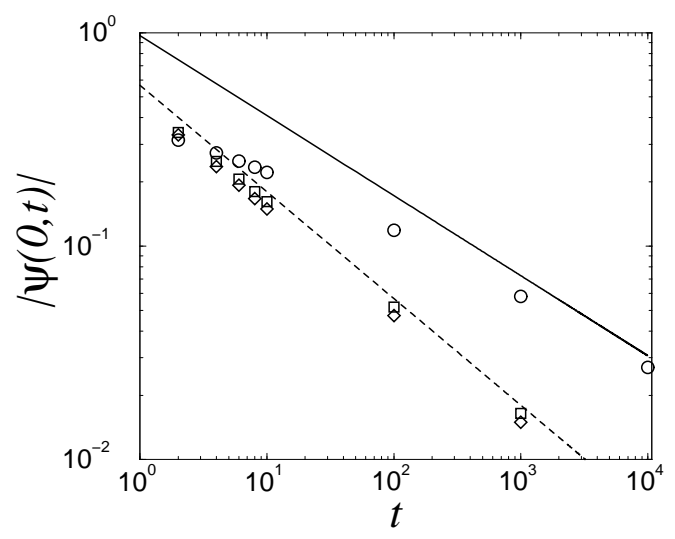

FIG. 2. Log-log plot of the time evolution of the wave packet amplitude at $x=0$ for three different wave packets. The usual asymptotic behavior $t^{-1 / 2}$ is observed for the Gaussian (diamond) and for the PLTWP defined by Eq. (6) with $\alpha=3$ (square). The anomalous decay predicted for PLTWP with $1 / 2<\alpha<1$ is observed for the PLTWP defined by Eq. (6) with $\alpha=0.75$ (circle). To estimate the degree of convergence, we also show the asymptotic behaviors predicted in the two cases as straight lines. The dashed line indicates the $t^{-1 / 2}$ behavior and the solid line shows the $t^{-0.375}$ behavior. In our calculation we set $\gamma=1, \hbar / 2 M=1$ and we chose the value of $\Delta x$ of the Gaussian wave packet equal to the same quantity of the PLTWP with $\alpha=3$ at $t=0$. 


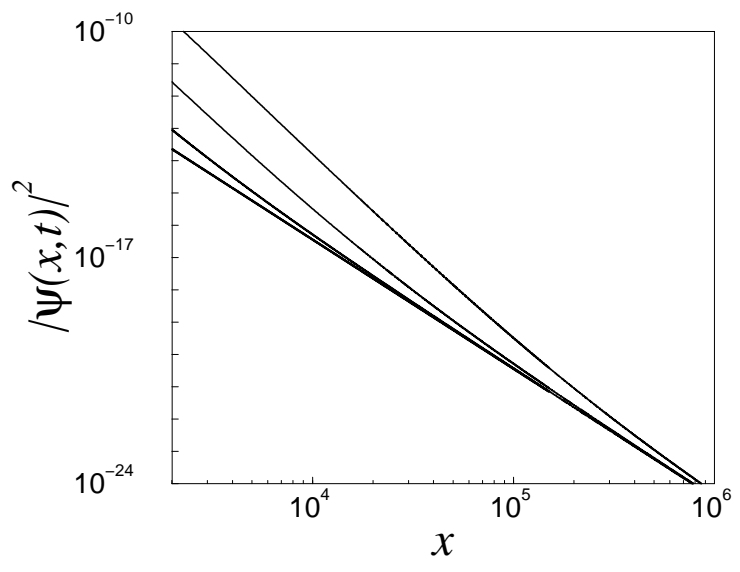

FIG. 3. Log-log plot of the positive tail of $|\psi(x, t)|^{2}$ for the PLTWP of Eq. (6) with $\alpha=2$ as a function of $x$ for different values of the time. The bottom line refers to the case $t=0$. From bottom to top the other lines describe the cases $t=10^{4}, t=10^{5}$, and $t=10^{6}$ respectively. The dominance of the leading term of the asymptotic expansion at $t=0$ is also observed for longer times at larger values of $x$. In our calculation we set $\gamma=1$ and $\hbar / 2 M=1$. 